\title{
Renoprotective Effects of Total Glucosides from Paeony against Nephrotoxicity Induced by Total Alkaloids from Semen Strychni
}

\author{
Mingming Lv, ${ }^{1}$ Meiyu Zhang, ${ }^{2}$ Yezhe Cheng, ${ }^{2}$ Kexia Zhang, \\ Chenzhi Hou, ${ }^{1}$ and Xiaohui Chen ${ }^{1}$ \\ ${ }^{1}$ School of Pharmacy, Shenyang Pharmaceutical University, Shenyang 110016, China \\ ${ }^{2}$ School of Traditional Chinese Materia Medica, Shenyang Pharmaceutical University, Shenyang 110016, China \\ Correspondence should be addressed to Xiaohui Chen; cxh_syphu@hotmail.com
}

Received 1 May 2017; Revised 18 July 2017; Accepted 2 August 2017; Published 22 October 2017

Academic Editor: Caigan Du

Copyright (C) 2017 Mingming Lv et al. This is an open access article distributed under the Creative Commons Attribution License, which permits unrestricted use, distribution, and reproduction in any medium, provided the original work is properly cited.

Semen Strychni have been shown to have therapeutic effect in improving blood circulation, relieving rheumatic pain, and treating cancer. However, Semen Strychni could cause severe nephrotoxicity. The present study was designed to evaluate whether treatment with total glucosides from paeony (TGP) has renoprotective effect against nephrotoxicity induced by total alkaloids from Semen Strychni (TAS). The levels of blood urea nitrogen (BUN) and creatinine (Cr) were determined and histopathological changes were also examined to evaluate renal injury. Moreover, a HPLC-MS method was developed and validated to investigate the comparative toxicokinetics of strychnine and brucine in rats plasma after oral administration of TAS and pretreatment with TGP. Results demonstrated that the levels of BUN and Cr were significantly increased $(p<0.05)$ in TAS group, together with tubule epithelium cloudy swelling, degeneration, and glomerular atrophy in rats' kidneys. The TAS-induced kidney damage was alleviated after pretreatment with TGP. Besides, $T_{\max }$ of strychnine and brucine were increased and $T_{1 / 2}$ of strychnine and brucine were decreased after pretreatment with TGP. The toxicokinetics study showed that pretreatment with TGP could attenuate the absorption of strychnine and brucine, as well as accelerate their elimination. These results suggest that TGP possesses renoprotective effects.

\section{Introduction}

Semen Strychni is a traditional Chinese herb, mainly used to treat rheumatoid arthritis, nervous disease, and traumatic pains and to promote blood circulation and remove blood stasis [1-3]. Total alkaloid from Semen Strychni (TAS) is a concentrated bioactive extract of Semen Strychni, which mainly consists of strychnine and brucine $[4,5]$. Strychnine and brucine possess analgesic, anti-inflammatory, and antitumor effects as well as toxicity $[6,7]$. The use of Semen Strychni was limited for its extreme toxicity, and it can also cause cardiac arrest, epileptic seizures, and nephrotoxicity $[8,9]$. The suggested therapeutic dose of Semen Strychni is $0.3-0.6 \mathrm{mg} /$ per day and the dose of its prime toxic ingredientstrychnine is $5-10 \mathrm{mg}$ [10-12]. In previous study, Semen Strychni has obvious renal injury [13]. Thus, it is necessary to reduce the nephrotoxicity of Semen Strychni.
Some research showed that combining Semen Strychni with Paeonia lactiflora could reduce the content of strychnine and brucine, increase the therapeutic effect, and reduce the toxicity of Semen Strychni $[14,15]$. Semen Strychni and Paeonia lactiflora have been compatibly used in many proprietary Chinese medicines such as Gujinwan Capsule, Huoxuezhitong Paste, and Huoluozhenfeng Pill to treat osteopenia and arthralgia and relieve pain. Total glucosides of paeony (TGP) are the predominate effective ingredients of the traditional Chinese herb, Paeonia alba radix (roots of Paeonia lactiflora Pall.), which mainly contains paeoniflorin and albiflorin. It was reported that TGP possesses analgesic, anti-inflammatory, and immunoregulatory effects $[16,17]$ and it was exerted renoprotection with antioxidative injury, preventing renal tubulointerstitial injury and blocking TLRs action $[18,19]$. It was also reported that TGP plays therapeutic role in experimental diabetic nephropathy [10]; moreover, 


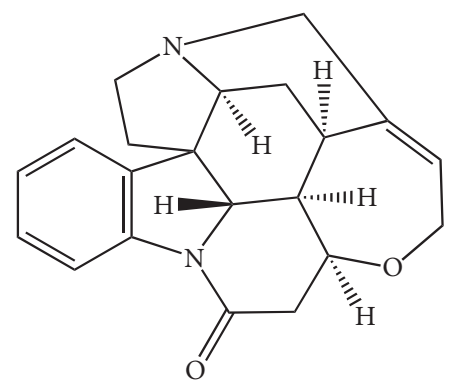

(a)

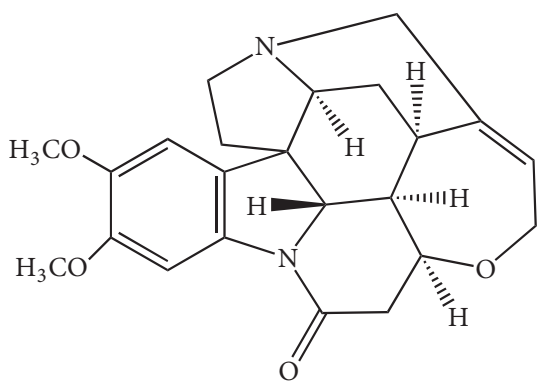

(b)

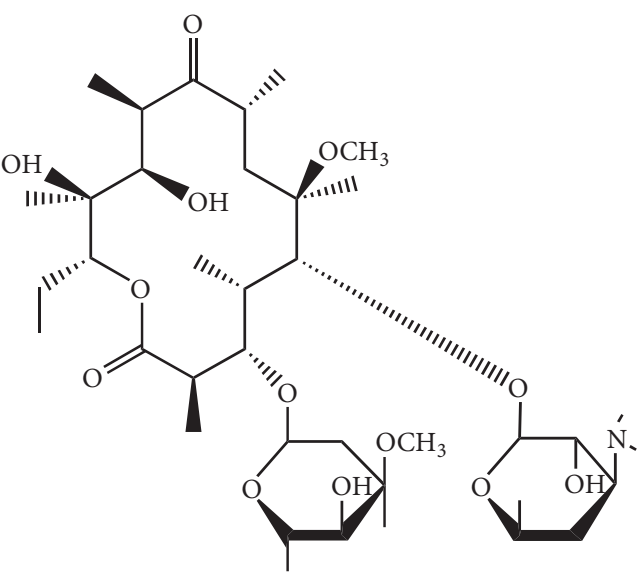

(c)

FIgURE 1: Chemical structures of strychnine (a), brucine (b), and IS clarithromycin (c).

TGP has already been used to treat chronic nephritis in clinic [20].

As far as we know, few investigation were developed to verify the effects of TGP on nephrotoxicity induced by TAS. The present study was designed to evaluate whether treatment with total glucosides from paeony exerts renoprotective effect against nephrotoxicity induced by total alkaloids from Semen Strychni. The levels of serum BUN, Cr, and kidney histopathological changes were used to certify the nephrotoxicity of TAS. What is more, the antidotal effects of TGP on the toxicokinetics of strychnine and brucine in rats were studied.

\section{Materials and Methods}

2.1. Material and Regents. Semen Strychni, Paeonia lactiflora Pall, and Aristolochia manshuriensis (AM) were purchased from Anguo Lengbei Co., Ltd (Hebei, China). All herbs were authenticated by Professor Ying Jia (Pharmacognosy Department, Shenyang Pharmaceutical University). The reference standards of strychnine and brucine were obtained from National Institute for control of Pharmaceutical and Biological Products (Beijing, China). Clarithromycin as internal standard (IS) was provided by Sigma-Aldrich (MO, USA) (Figure 1). The purity of each reference standard was all above 98\%. HPLC grade methanol and acetonitrile were purchased from Fisher Scientific (Nanjing, China). HPLC Glacial acetic acid was obtained from Yuwang Industry Co. Ltd. (Shandong, China). Distilled water, prepared from demineralized water, was used throughout experiment.

2.2. Herb Preparation. The dried seeds of Semen Strychni $(100 \mathrm{~g})$ were extracted three times by refluxing with $1 \mathrm{~L}$ of $70 \%$ ethanol $(1: 10 \mathrm{~m} / \mathrm{v})$ for $1 \mathrm{~h}$ each time. The collected solvent was concentrated under rotary evaporation to remove all ethanol. The residue was suspended into water and acidified to $\mathrm{pH}=$ 2 with $\mathrm{HCl}(1.0 \mathrm{~mol} / \mathrm{L})$ and then extracted with methylene chloride. The $\mathrm{pH}$ of aqueous layer was adjusted to 12 with $4.0 \mathrm{~mol} / \mathrm{L} \mathrm{NaOH}$ and then extracted with methylene chloride once more. The methylene chloride layer was concentrated under rotary evaporation to remove all ethanol, and the resultant residue was the TAS extract. The concentrations of strychnine and brucine were determined to be $33.1 \%$ and 25.9\% by HPLC method.

The powder of Paeonia lactiflora Pall radix (250 g) was extracted three times by refluxing with $2 \mathrm{~L}$ of $70 \%$ ethanol $(1: 8, \mathrm{~m} / \mathrm{v})$ for $2 \mathrm{~h}$ each time. The filtrates were concentrated by removing all ethanol solvent in rotary evaporation. The residues were suspended into water $(0.2 \mathrm{~g} / \mathrm{mL})$. The sample solution flowed through glass column (equipped with AB8 resign). During adsorptive equilibrium, the adsorb-laden column was washed with water firstly and then desorbed with $70 \%$ ethanol. The collected solution (70\% ethanol) was concentrated by rotary evaporation and the residue was the TGP extract.

Dried stem (100 g) of Aristolochia manshuriensis was extracted three times by refluxing with $400 \mathrm{~mL}$ of water $(1: 4, \mathrm{~m} / \mathrm{v})$ for $2 \mathrm{~h}$ each time. The obtained solutions were concentrated by rotary evaporation and the residue was the AM extract.

All extracts were redissolved with carboxymethylcellulose sodium (CMC-Na, 0.5\%) and diluted to an appropriate volume.

2.3. Animal Treatment. Ninety-six male Sprague-Dawley rats $(250 \pm 20 \mathrm{~g})$ were kindly supplied by the Experimental Animal Central of Shenyang Pharmaceutical University. All animal-use procedures were in accordance with the regulations for animal experimentation issued by the State Committee of Science and Technology of the People's Republic of China. Animals were housed in laboratory conditions and acclimatized to 7 days before experiments.

Eighty rats were used to access the renal injury induced by TAS and randomly divided into four groups ( $n=20 /$ group): blank group, positive control group, TGP group, and TAS group. Rats in blank group, positive control group, and TAS group were orally given the approximately same volume of $0.5 \%$ CMC-Na solution for 7 days. Rats in TGP group were 
orally administrated with TGP $(280 \mathrm{mg} / \mathrm{kg})$ for 7 days. At the 8 th days, rats were orally administrated with $0.5 \% \mathrm{CMC}-\mathrm{Na}$ solution (for the blank group), AM (5.25 g/ $\mathrm{kg}$ for the positive control group), and TAS $(6.5 \mathrm{mg} / \mathrm{kg}$ for the TGP and TAS group), respectively.

Blood samples were taken from the abdominal aorta at $0.16,0.33,0.5,1,4,9$, and $24 \mathrm{~h}$ after the final administration on 8 th day, and serum was obtained by centrifugation of blood $(4,000 \mathrm{rpm}$ for $5 \mathrm{~min})$. Rats $(n=2$ for each group, 56 in total) were separately sacrificed at $0.16,0.33,0.5,1,4,9$, and $24 \mathrm{~h}$ after the final administration on 8th day, and the kidney tissues were excised and fixed in 10\% formalin-saline for at least $24 \mathrm{~h}$ and then embedded in paraffin.

Other male SD rats $(250 \pm 20 \mathrm{~g})$ were used in this part of study. Rats were randomly divided into two groups $(n=$ 8), namely, TAS group and TGP group. Before TAS was administrated to rats in the two groups on 8 th, TGP group and TAS group were orally given TGP at a dose of $280 \mathrm{mg} / \mathrm{kg}$ and $0.5 \%$ CMC-Na solution for 7 days, respectively. After oral administration of TAS at a dose of $6.5 \mathrm{mg} / \mathrm{kg}$ on 8 th day, an aliquot of $300 \mu \mathrm{L}$ blood samples was taken from the eye ground veins at $0.03,0.08,0.16,0.33,0.75,1,2,4,6$, and $9 \mathrm{~h}$ after oral administration and then centrifuged at 13,000 rpm for $5 \mathrm{~min}$. Plasma samples were collected and stored at $-20^{\circ} \mathrm{C}$ until analysis.

2.4. Serum BUN, Cr, and Histopathology. The levels of BUN and $\mathrm{Cr}$ in serum were assayed by kits. Kidney samples were sliced into $3 \mu \mathrm{m}$ wax sections, and the sections were stained with hematoxylin-eosin (H\&E) and examined under a light microscope (OLYMPUS, DP72, Japan).

2.5. Instruments and LC-MS Conditions. The analysis was performed on a Shimadzu (Japan) LC-MS 2010 EV system equipped with an electrospray ionization (ESI) interface. The liquid chromatographic separation was achieved on CAPCELL PAK $\mathrm{C}_{18}$ column $(150 \mathrm{~mm} \times 4.5 \mathrm{~mm}, 5 \mu \mathrm{m})$, which was protected by a guard column $(4 \mathrm{~mm} \times 2 \mathrm{~mm}, 5 \mu \mathrm{m})$. The mobile phase, consisting of acetonitrile (solvent A) and $0.05 \%$ glacial acetic acid water (Solvent B), was delivered at a flow rate of $0.8 \mathrm{~mL} / \mathrm{min}$ with $25 \%$ of the eluent split into the inlet of mass spectrometer. The gradient program was shown as follows: $0-1 \mathrm{~min}, 13-25 \% \mathrm{~A} ; 1-3 \mathrm{~min}, 25 \% \mathrm{~A} ; 3-5 \mathrm{~min}, 25-80 \%$ A; 5-7 min, $80 \%$ A; 7-8 $\min , 80-13 \%$ A; 8-10 min, $13 \%$ A. The column and autosampler tray temperature were maintained constantly at $35^{\circ} \mathrm{C}$ and $4^{\circ} \mathrm{C}$, respectively. The inject volume was $10 \mu \mathrm{L}$ and the run time was $10 \mathrm{~min}$.

The analytes and IS were ionized by ESI source in positive ion mode under the following source conditions: nebulizing gas $1.5 \mathrm{~L} / \mathrm{min}$, curved desolvation line (CDL) temperature $250^{\circ} \mathrm{C}$, heat block temperature $250^{\circ} \mathrm{C}$, detector voltage $1.95 \mathrm{kV}$, and the other parameters were fixed as tuning file. Selected ion monitoring (SIM) mode was applied for the quantification of $[\mathrm{M}+\mathrm{H}]^{+}$at $m / z 335.05$ for strychnine, [M $+\mathrm{H}]^{+}$at $m / z 395.10$ for brucine, and $[\mathrm{M}+\mathrm{H}]^{+}$at $m / z 748.45$ for IS, respectively.

2.6. Preparation of Calibration Standards and Quality Control Samples. Reference substances of strychnine, brucine, and
IS were accurately weighted and dissolved in acetonitrile to get standard stock solutions $(200 \mu \mathrm{g} / \mathrm{mL}$ for strychnine, $100 \mu \mathrm{g} / \mathrm{mL}$ for brucine, and $100 \mu \mathrm{g} / \mathrm{mL}$ for IS, resp.). A series of working solutions were prepared by diluting the stock solution with acetonitrile to get the final concentration (5-400 ng/mL for strychnine, 5-100 ng/mL for brucine, and $1000 \mathrm{ng} / \mathrm{mL}$ for IS). Quality control (QC) solutions in plasma were prepared in the same way at low, middle, and high concentrations: 15,100 , and $320 \mathrm{ng} / \mathrm{mL}$ for strychnine; and 15 , 50 , and $80 \mathrm{ng} / \mathrm{mL}$ for brucine. All solutions were stocked at $4^{\circ} \mathrm{C}$.

2.7. Plasma Sample Preparation. An aliquot of $100 \mu \mathrm{L}$ plasma was spiked with $20 \mu \mathrm{L}$ of IS, followed by adding $300 \mu \mathrm{L}$ acetonitrile. The mixed solutions were vortexed for $3 \mathrm{~min}$ and centrifuged at 13,000 rpm for $5 \mathrm{~min}$; then the supernatant was transferred into a clean centrifuge tube and dried under a stream of nitrogen. The residue was reconstituted with $100 \mu \mathrm{L}$ of initial mobile phase and vortexing for $1 \mathrm{~min}$, followed by centrifuging for another $10 \mathrm{~min}$. Finally, an aliquot of $10 \mu \mathrm{L}$ of the supernatant was injected into the HPLC-MS system for analysis.

2.8. HPLC-MS Method Validation. In our study, the HPLCMS method was validated in accordance with FDA guidelines $[21,22]$, including selectivity, linearity, lower limited of quantification (LLOQ), precision, accuracy, extraction recovery, matrix effect, carry-over, and stability.

Selectivity was accessed by comparing chromatograms of blank plasmas obtained from six different SD rats, blank plasma sample spiked with analytes and IS, and plasma obtained $0.33 \mathrm{~h}$ after oral administrated of TAS.

The linearity of the assay was assessed by analyzing the calibration curves using least-squares linear regression of the peak area of the analytes to IS versus the nominal concentration of the calibration standard with a weighted factor $\left(1 / C^{2}\right)$. The LLOQ was defined as the lowest concentration on calibration curve with an acceptable accuracy (between 80 and $120 \%$ ) and the precision below $20 \%$.

Accuracy and precision (inter- and intraday) were measured by determining QC samples at three concentrations levels with 6 replicates on three consecutive validation days. Accuracy was evaluated as relative error (RE\%) and precision was evaluated as relative standard deviation (RSD\%).

Recovery was calculated by comparing peak areas from extracted samples with those postextracted samples spiked with analytes and IS $(A)$. Matrix effect was calculated by calculating the peak area ratios of $A$ to pure standard solution containing equivalent amounts of the compounds. Both were performed at three QC levels.

Stability studies in plasma samples were also conducted at three QC levels in several different storage conditions: at room temperature for $12 \mathrm{~h}$, at $-20^{\circ} \mathrm{C}$ for at least $15 \mathrm{~d}$, after three freeze-thaw cycles, and $12 \mathrm{~h}$ after preparing at $4^{\circ} \mathrm{C}$.

Carry-over was assessed by injecting two blank sample extracts after upper limit quantification (ULOQ), and peak area found in first blank sample should be less than $20 \%$ of the peak area at LLOQ of analytes. 


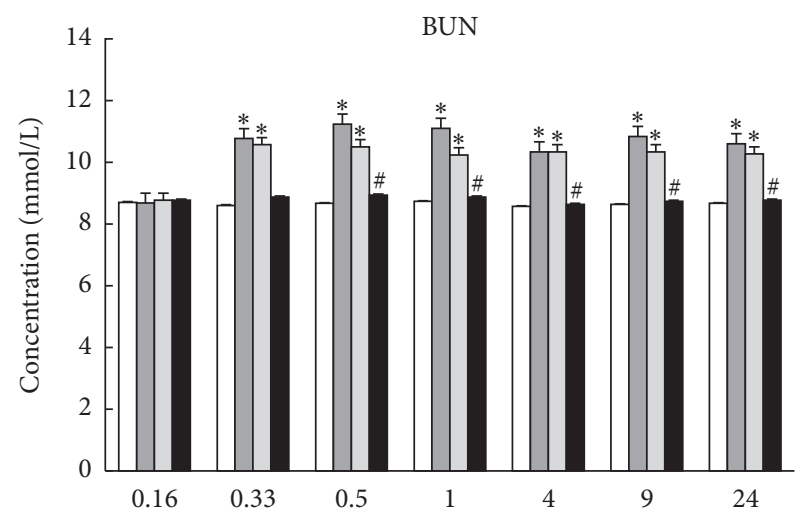

(h)

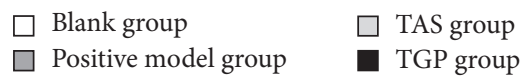

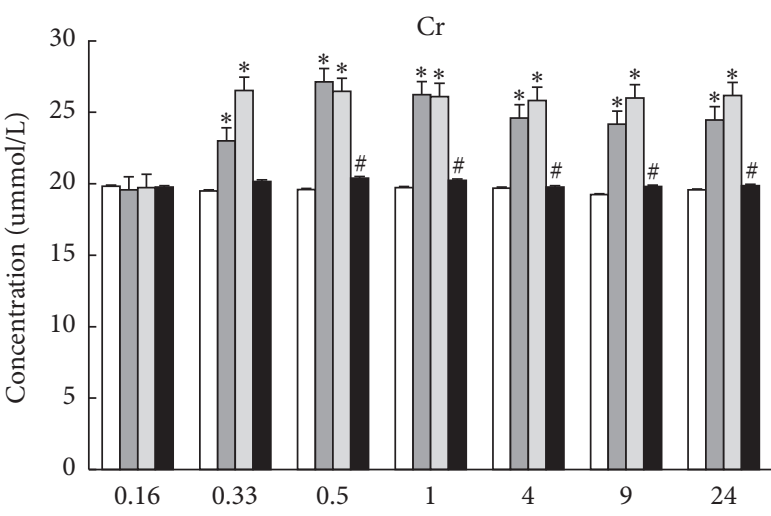

(h)

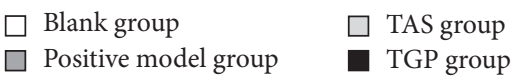

Figure 2: The levels of serum BUN and Cr of four groups on different time points. All values are indicated as mean $\pm \operatorname{SD}(n=8)$. ${ }^{*} p<0.05$ significant difference between other groups and blank group. ${ }^{\#} p<0.05$ significant difference between TGP group and TAS group.

2.9. Statistical Analysis. The concentration of the analytes was calculated by the corresponding calibration curve. Toxicokinetics studies were processed by DAS 2.1.1 software package (Chinese Pharmacological Society) to calculate half-life $\left(T_{1 / 2}\right)$, the maximum plasma concentration $\left(C_{\max }\right)$, the corresponding time $\left(T_{\max }\right)$, and the area under concentrationtime curve (AUC). Results of renal function markets and the toxicokinetics parameters were expressed as mean \pm standard deviation (mean $\pm \mathrm{SD}$ ). Statistical analysis was performed using SPSS 19.0 software package by one-way analysis of variance (ANOVA) followed by Tukey-Kramer multiple comparison tests. Statistical significance was considered when the value of $p<0.05$.

\section{Results and Discussion}

3.1. Assessment of Renal Function Markers and Analysis. Experimental and clinical studies have demonstrated that renal damage usually is accompanied by serum biochemical changes. For example, patients with acute renal failure usually have higher BUN and Cr $[23,24]$. Increased level of serum BUN indicated the weaker ability of glomerular filtration rate and the loss of renal tubular reabsorption selectivity in acute and chronic nephritis. Moreover, increased level of serum $\mathrm{Cr}$ indicates that renal excretion has been severely damaged $[25,26]$. The results of serum BUN and Cr in this study are presented in Figure 2. In comparison with the blank group, levels of BUN and $\mathrm{Cr}$ in serum were significantly increased $(p<0.05)$ after the final administration on 8th day from $0.16 \mathrm{~h}$ to $24 \mathrm{~h}$ in the TAS and positive control group, which indicated that kidney was damaged. The levels of serum BUN and Cr in TGP group showed no significant difference comparing to blank group, but remarkably decreased $(p<$ 0.05 ) from $0.16 \mathrm{~h}$ to $24 \mathrm{~h}$ comparing to TAS group. This result suggested that TGP could attenuate the nephrotoxicity caused by TAS.
3.2. Histopathology. Histopathological changes were examined to investigate TAS-induced nephrotoxicity, which can directly help us understand the histopathological changes in kidney. Histopathological changes in the rat kidney are presented in Figure 3. Figure 3 (A1-A7) shows no abnormal morphological changes in the kidney specimen in the rats of blank group. Rats in positive group (Figure 3, B2-B7) and TAS group (Figure 3, C2-C7) show that the kidney sections gradually presented tubular epithelium cloudy swelling, degeneration, and glomerular atrophy. No abnormal morphological changes appeared in TGP group rats (Figure 3, D1-D7). Histopathology study demonstrated that TGP could protect kidney against TAS-induced renal injury, which is supported by the results of renal function biomarkers. The reasons of renal function markers and histopathology changes might be explained as TGP could inhibit macrophage infiltration into kidney, resist renal inflammation, and renal tubular epithelial cell transdifferentiation and exert antioxidative stress effect $[18,27]$. The results of renal function biomarkers and histopathology changes suggested that TGP might display renoprotective effects on nephrotoxicity induced by TAS.

3.3. HPLC-MS Method Validation. No interfering peak from endogenous substances was observed in the representative chromatogram of blank plasma sample at the retention time of the analytes and IS. The retention times of strychnine, brucine, and IS were 3.82, 3.90, and $6.20 \mathrm{~min}$, respectively (Figure 4). The calibration curves showed good linearity in the range of $5-400 \mathrm{ng} / \mathrm{mL}$ for strychnine and $5-100 \mathrm{ng} / \mathrm{mL}$ for brucine. Typical regression equations of the calibration curves were $y=0.0180 x+0.0093(r=0.9961)$ for strychnine and $y=0.0120 x+0.0062(r=0.9987)$ for brucine. The LLOQs of strychnine and brucine were $5 \mathrm{ng} / \mathrm{mL}$ with the RSD\% less than $10.2 \%$, which was within acceptable limits. Precision and accuracy were satisfactory at three levels of QC samples. The extraction solvent used in experiment showed 

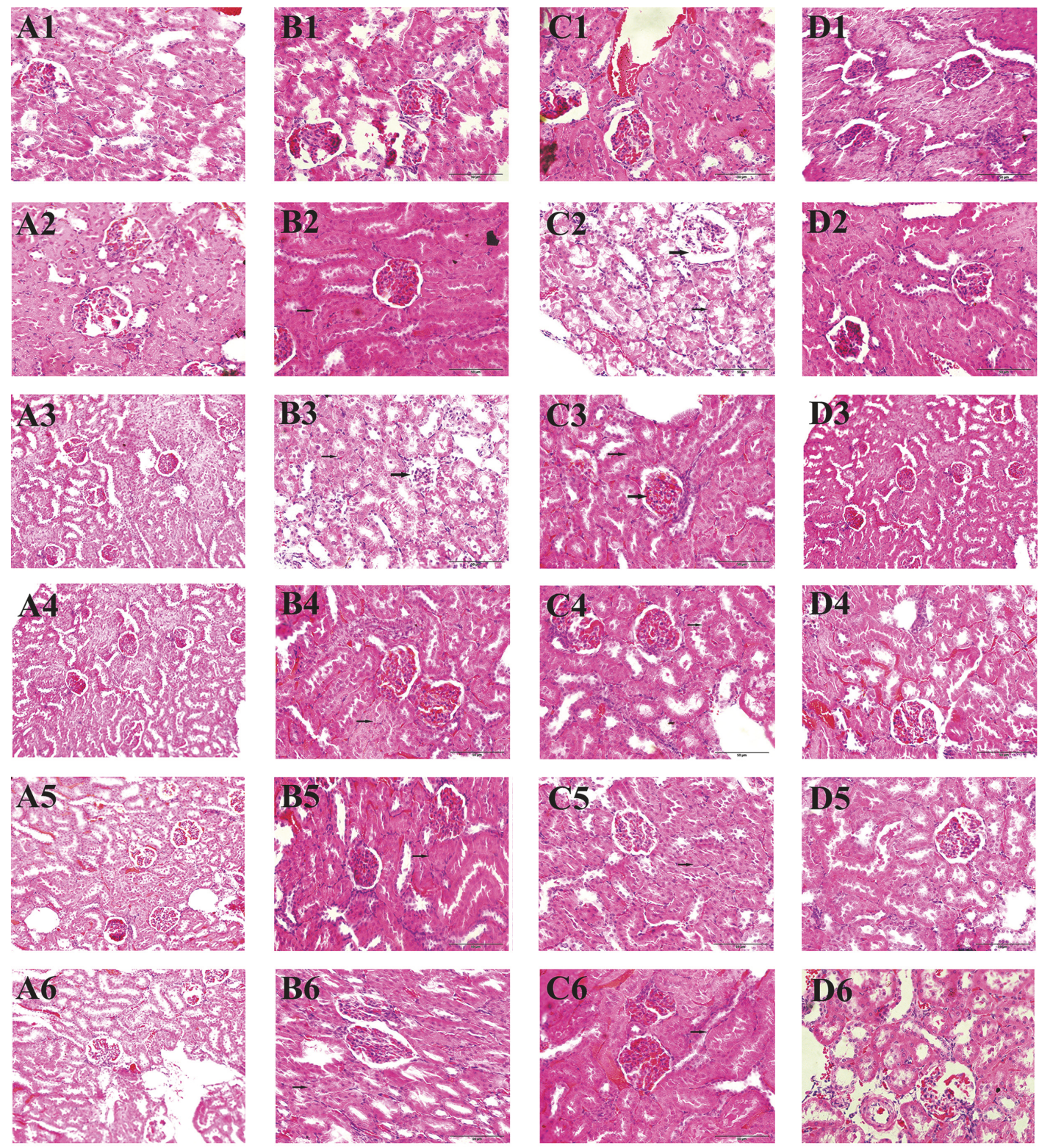

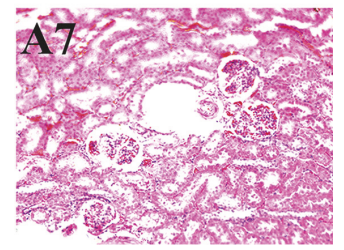

(a)

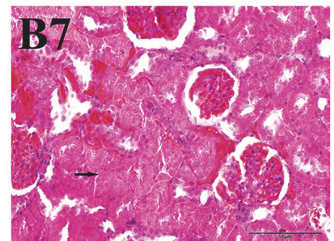

(b)

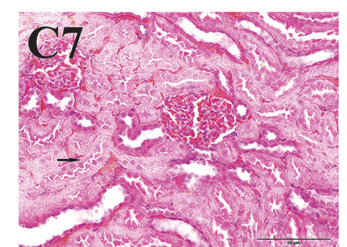

(c)

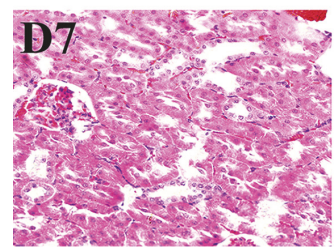

(d)

FIGURE 3: Representative histopathological photographs of SD rats' kidney sections $(\times 100)$ in different groups. Rats in blank group (a), rats in positive control group (b), rats in TAS group (c), rats in TGP group (d). 1-7: kidney condition at 0.16, 0.33, 0.5, 1, 4, 9, and $12 \mathrm{~h}$ (different kinds of pathology lesions were marked by different arrows).

good extraction recovery. The recoveries of strychnine and brucine in three QC concentrations were $81.1-91.9 \%$ and $82.2-92.2 \%$, and the recovery of IS was $95.5 \%$. The results of matrix effect were $88.3-93.4 \%$ for strychnine and $87.5-104.6 \%$ for brucine, and the matrix effect of IS was $98.9 \%$. The analytes were stable (RE\% within $11.4 \%$ and RSD\% below 9.1\%) under tested conditions and no carry-over was found for the method (showed in Table 1). The results indicated that the developed method was reliable and reproducible to quantitate strychnine and brucine in rat plasma. 

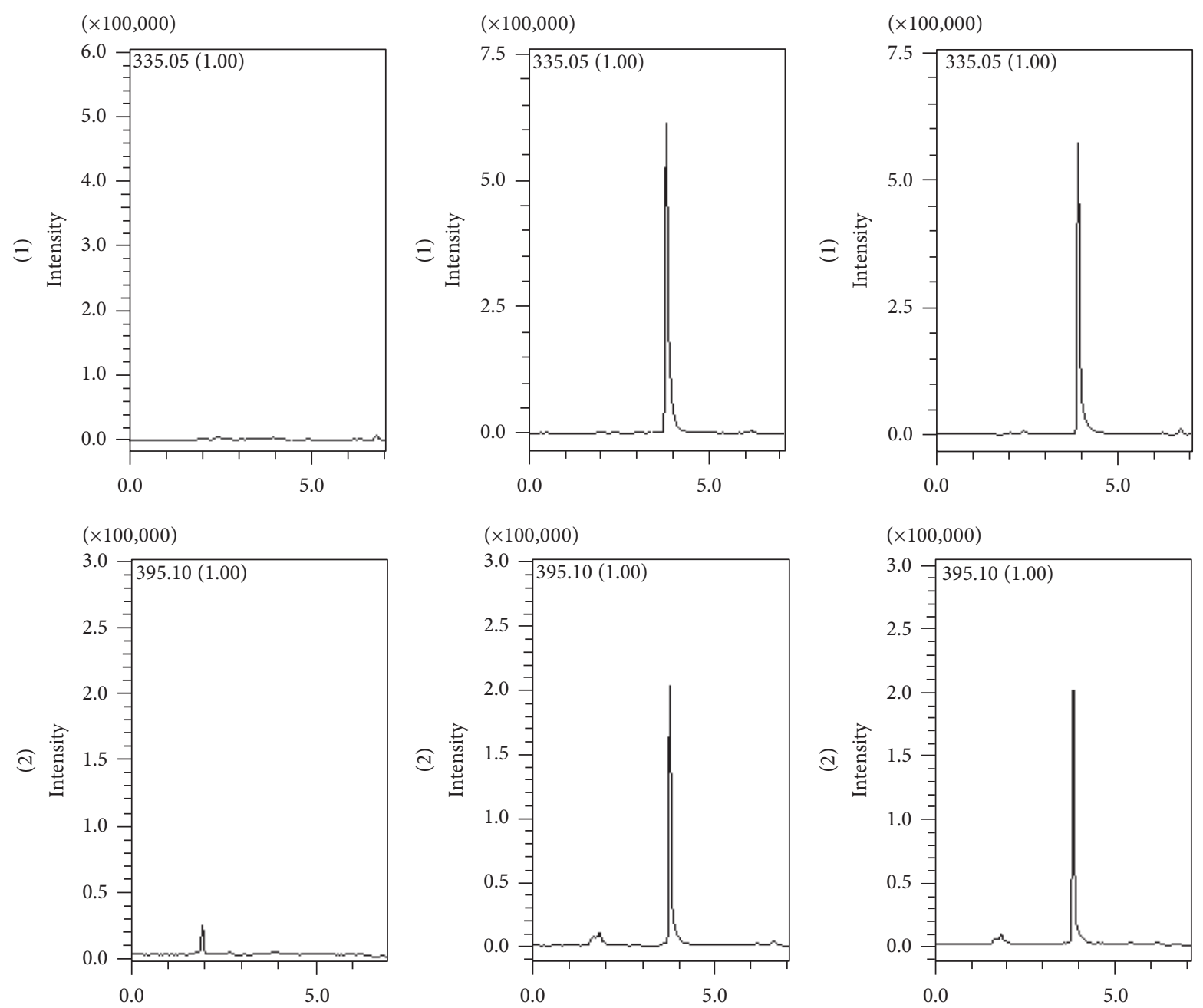

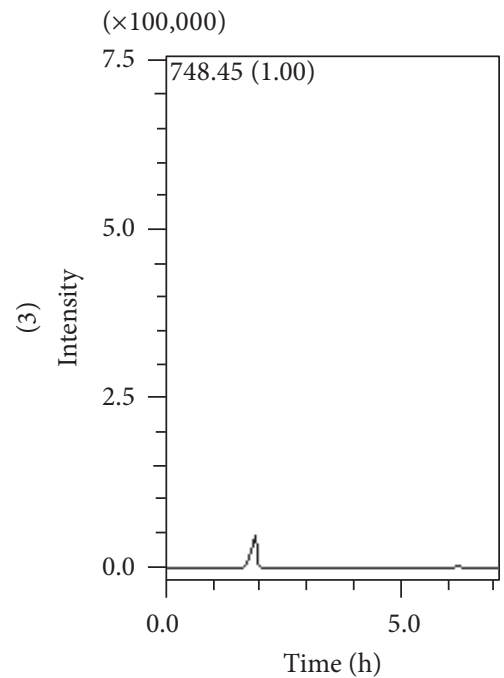

(a)

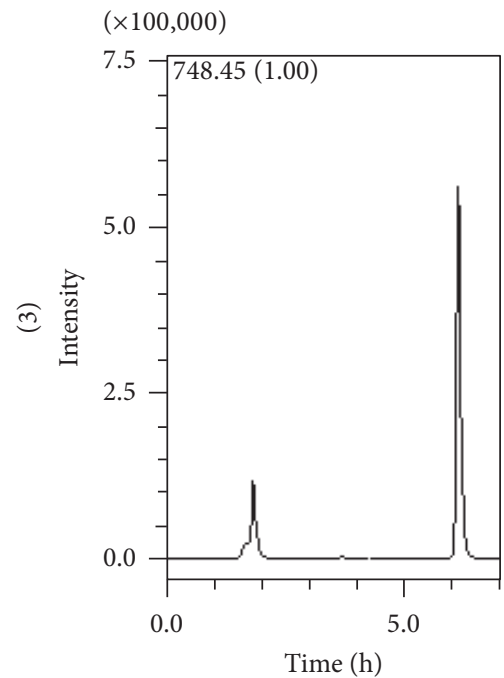

(b)

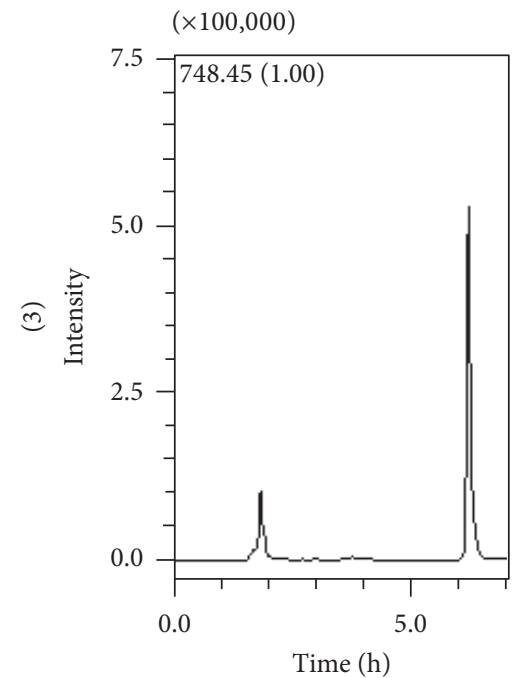

(c)

FIGURE 4: Typical chromatograms of strychnine (1), brucine (2), and IS (3). (a) Blank plasma, (b) blank plasma with strychnine, brucine, and IS, and (c) plasma sample obtained $0.33 \mathrm{~h}$ after oral administration of TAS extract. 
TABLE 1: Precision, accuracy, recovery, and matrix effect of HPLC-MS method for analysis of analytes and IS $(n=8)$.

\begin{tabular}{lcccccc}
\hline Analytes & $C(\mathrm{ng} / \mathrm{mL})$ & $\begin{array}{c}\text { Intraday } \\
(\mathrm{RSD} \%)\end{array}$ & $\begin{array}{c}\text { Interday } \\
(\mathrm{RSD} \%)\end{array}$ & $\begin{array}{c}\text { Accuracy } \\
(\mathrm{RE} \%)\end{array}$ & $\begin{array}{c}\text { Recovery } \\
(\%, \text { mean } \pm \mathrm{SD})\end{array}$ & $\begin{array}{c}\text { Matrix effect } \\
(\%, \mathrm{mean} \pm \mathrm{SD})\end{array}$ \\
\hline \multirow{3}{*}{ Strychnine } & 15 & 13.0 & 10.3 & -15.4 & $81.1 \pm 5.6$ & $92.5 \pm 6.8$ \\
& 140 & 7.5 & 7.9 & 9.1 & $95.4 \pm 4.3$ & $93.4 \pm 6.4$ \\
& 300 & 4.6 & 6.5 & 4.8 & $91.9 \pm 5.3$ & $88.3 \pm 5.6$ \\
Brucine & 15 & 11.8 & 10.0 & -14.7 & $82.2 \pm 12.6$ & $97.5 \pm 6.4$ \\
& 50 & 6.1 & 5.3 & 3.5 & $91.8 \pm 4.7$ & $104.6 \pm 5.2$ \\
& 80 & 3.6 & 4.9 & 3.2 & $99.3 \pm 4.2$ \\
\hline
\end{tabular}

TABLE 2: Toxicokinetics parameters of strychnine and brucine in rat plasma after oral administration of TAS extract (mean \pm SD, $n=8)$.

\begin{tabular}{lcccc}
\hline Parameters & \multicolumn{2}{c}{ Strychnine } & \multicolumn{2}{c}{ Brucine } \\
& TAS group & TGP group & TAS group & TGP group \\
\hline$T_{1 / 2}(\mathrm{~h})$ & $2.5 \pm 0.5$ & $1.4 \pm 0.3^{*}$ & $2.7 \pm 0.4$ & $1.1 \pm 0.2^{*}$ \\
$T_{\max }(\mathrm{h})$ & $0.3 \pm 0.2$ & $0.5 \pm 0.1^{*}$ & $0.2 \pm 0.1$ & $0.3 \pm 0.2^{*}$ \\
$C_{\max }(\mathrm{ng} / \mathrm{mL})$ & $172.6 \pm 28.2$ & $153.1 \pm 16.3$ & $40.5 \pm 2.8$ & $28.1 \pm 4.3^{*}$ \\
$\mathrm{AUC}_{0-t}(\mathrm{ng} \mathrm{h} / \mathrm{mL})$ & $308.9 \pm 76.2$ & $258.8 \pm 53.8$ & $79.2 \pm 8.1$ & $42.0 \pm 5.0^{*}$ \\
$\mathrm{AUC}_{0-\infty}(\mathrm{ng} \mathrm{h} / \mathrm{mL})$ & $330.1 \pm 80.8$ & $275.6 \pm 64.9$ & $99.7 \pm 13.9$ & $45.1 \pm 5.6^{*}$ \\
\hline
\end{tabular}

${ }^{*} p<0.05$ significant differences between TAS group and TGP group.

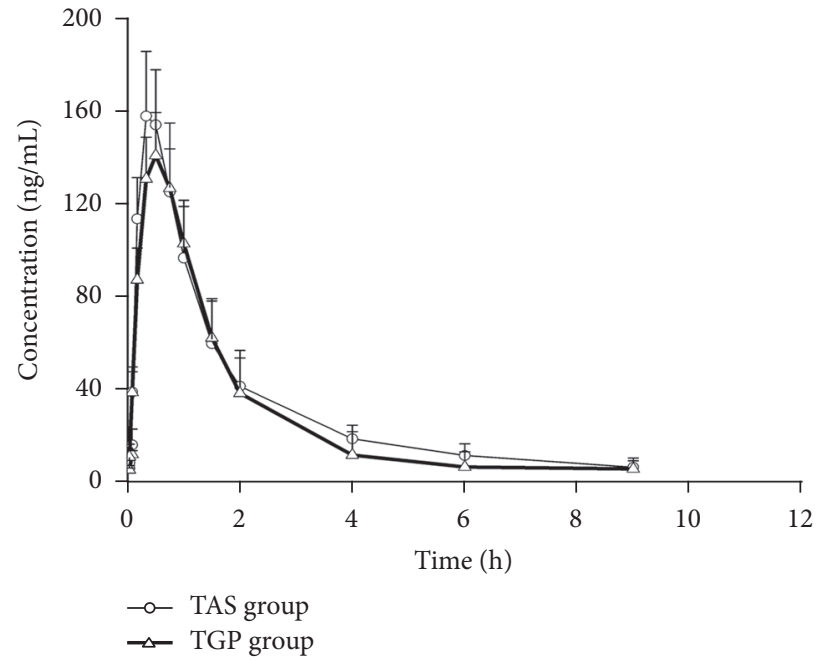

(a)

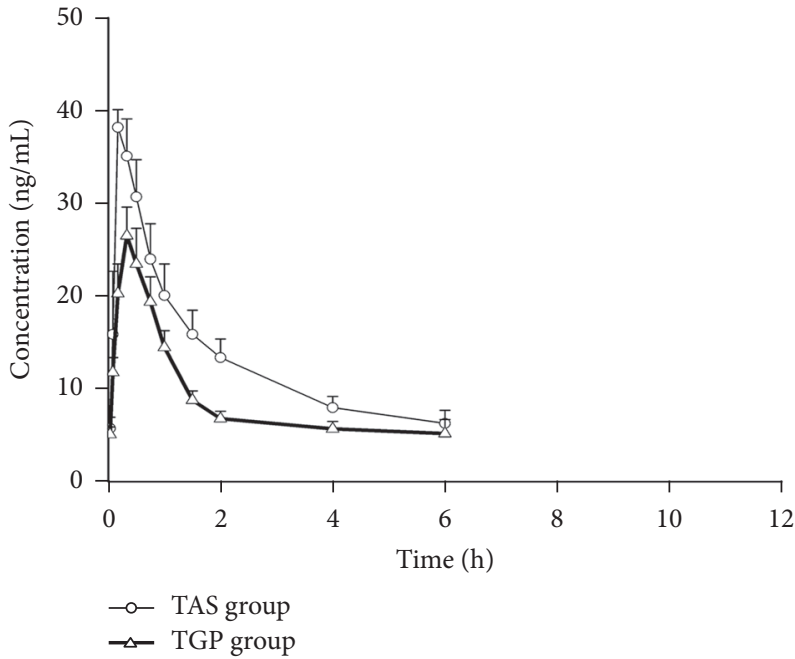

(b)

FIGURE 5: Mean plasma concentration-time curves of strychnine (a) and brucine (b) in rat plasma after oral administration of TAS extract at a dose of $6.5 \mathrm{mg} / \mathrm{kg}(n=8)$.

3.4. Toxicokinetics Results. The validation method was successfully applied to the toxicokinetics of strychnine and brucine in rat plasma after oral administration of TAS. The concentration-time profiles of TAS group and TGP group are illustrated in Figure 5, and the corresponding toxicokinetics parameters are summarized in Table 2.

Oral administration of TAS in rat can cause obvious nephrotoxicity, which is supported by biomarkers and pathological tests. The dose of TAS was verified according to previous study and based on our preliminary experiments [3]. AM was selected as nephrotoxic positive control herb for obvious nephrotoxicity [28]. Moreover, the dose of TGP was selected referring to the reports on TGP preventing renal damage in diabetic rats [18].

As is shown in Table 2, there were statistical differences in several toxicokinetics parameters including $T_{1 / 2}, T_{\max }, C_{\max }$, and AUC for analytes between TAS group and TGP group. Comparing with TAS group, $T_{1 / 2}$ of strychnine and brucine in TGP group significantly decreased $(p<0.05)$ and their $T_{\max }$ postponed $(p<0.05)$. The parameters of $C_{\max }$ and AUC decreased $(p<0.05)$ significantly in brucine and showed slight decrease $(p>0.05)$ in strychnine in TGP groups comparing to TAS group. These changes indicated that pretreatment of TGP could affect the toxicokinetic 
profiles of strychnine and brucine and attenuate the toxicity of TAS.

The toxicity of TAS was does-depended, and the decreased $C_{\max }$ and AUC in TGP group might contribute to preventing strychnine and brucine from the lethal does. Their increased $T_{\max }$ were also observed in TGP group, which may be caused by activating P-gp property of paeoniflorin [29]. The increase of $T_{\max }$ indicated that the delay of absorption of strychnine and brucine might contribute to the renoprotective effect of TGP.

In TGP group, the decreased $T_{1 / 2}$ of strychnine and brucine were observed, illustrating that TGP may have accelerated their elimination. Some researchers proved that strychnine and brucine are substrates of CYPs [30, 31]. Furthermore, paeoniflorin and albiflorin (main effective compounds of TGP) were reported to regulate the activity of CYP3A4 and CYP2D6 with varying degrees [32]. It illuminated that TGP induced the activity of CYP3A4 and CYP2D6, accelerated the metabolism of strychnine and brucine, and thus reduced the toxicity of TAS. It might be another proof to explain the decreased $T_{1 / 2}$ of strychnine and brucine and the renoprotective effect of TGP.

The preventive and therapeutic capacity of TGP against renal damage associated with anti-inflammatory and antioxidant effects might be another reason for TGP ameliorating nephrotoxicity induced by TAS [33,34]. All those results above indicated that TGP could protect the kidney against nephrotoxicity induced by TAS and attenuate the toxicity of Semen Strychni. This study supplied a guidance for the safe medication of Semen Strychni in clinic and was utilized in clinic for protecting kidney against nephrotoxicity induced by not only TAS but also other drugs.

\section{Conclusions}

In conclusion, this study indicated that pretreatment with TGP could recover the levels of BUN and $\mathrm{Cr}$, ameliorate kidney morphological changes, and thus exert renoprotective effect against nephrotoxicity induced by TAS. The toxicokinetics results of two groups showed that pretreatment with TGP could attenuate the absorption of strychnine and brucine and accelerate their elimination and may demonstrate the possible mechanism of TGP against nephrotoxicity induced by Semen Strychni. What is more, this study might be further utilized in clinic for nephrotoxicity induced by other drugs.

\section{Conflicts of Interest}

The authors declare that there are no conflicts of interest regarding the publication of this paper.

\section{Authors' Contributions}

Mingming Lv, Meiyu Zhang, and Xiaohui Chen conceived and designed the experiments; Mingming Lv, Meiyu Zhang, Yezhe Cheng, Kexia Zhang, and Chenzhi Hou performed the experiments; Mingming Lv, Meiyu Zhang, and Yezhe Cheng analyzed the data; Mingming Lv, Meiyu Zhang, and Xiaohui
Chen contributed to the reagents/materials/analysis tools; Meiyu Zhang wrote the paper.

\section{Acknowledgments}

This work was financially supported by Natural Science Foundation of China (no. 81673577).

\section{References}

[1] Chinese Pharmacopoeia Commission, Pharmacopoeia of the People's Republic of China, vol. 1, China Medical Science Press, 2010.

[2] S. Saraswati, A. A. Alhaider, and S. S. Agrawal, "Anticarcinogenic effect of brucine in diethylnitrosamine initiated and phenobarbital-promoted hepatocarcinogenesis in rats," Chemico-Biological Interactions, vol. 206, no. 2, pp. 214-221, 2013.

[3] S. Li, M. Zhang, P. Hou et al., "Identification of the toxic components in SemenStrychni and their metabolites in rat serum by high performance liquid chromatography coupled with a $\mathrm{Q}$ Exactive high-resolution benchtop quadrupole Orbitrap mass spectrometer," RSC Advances, vol. 95, no. 5, pp. 77689-77698, 2015.

[4] B. C. Cai, H. Wu, X. W. Yang et al., "Analysis of Spectral Data for ${ }^{13}$ CNMR of Sixteen Strychnos Alkaloids," Acta Pharmaceutica Sinica, vol. 29, 48 pages, 1994.

[5] G. Yang, Y. Pan, L. Liu, and Y. Jiang, "Simultaneous determination of strychnine, brucine, strychnine $\mathrm{N}$-oxide and brucine N-oxide in crude and fermented nux vomica by CZE with ephedrine hydrochloride as an internal standard," Chromatographia, vol. 73, no. 11-12, pp. 1223-1228, 2011.

[6] J.-X. Tian, C. Peng, L. Xu, Y. Tian, and Z.-J. Zhang, "In vitro metabolism study of Strychnos alkaloids using highperformance liquid chromatography combined with hybrid ion trap/time-of-flight mass spectrometry," Biomedical Chromatography, vol. 27, no. 6, pp. 775-783, 2013.

[7] X.-K. Deng, W. Yin, W.-D. Li et al., "The anti-tumor effects of alkaloids from the seeds of Strychnos nux-vomica on HepG2 cells and its possible mechanism," Journal of Ethnopharmacology, vol. 106, no. 2, pp. 179-186, 2006.

[8] J. A. Perper, "Fatal strychnine poisoning-A case report and review of the literature," Journal of Forensic Sciences, vol. 30, no. 4, pp. 1248-1255, 1985.

[9] G. Philippe, L. Angenot, M. Tits, and M. Frédérich, "About the toxicity of some Strychnos species and their alkaloids," Toxicon, vol. 44, no. 4, pp. 405-416, 2004.

[10] D. M. Wood, E. Webster, D. Martinez, P. I. Dargan, and A. L. Jones, "Case report: Survival after deliberate strychnine selfpoisoning, with toxicokinetic data," Critical Care, vol. 6, no. 5, pp. 456-459, 2002.

[11] C. Duverneuil, G. L. De La Grandmaison, P. De Mazancourt, and J.-C. Alvarez, "Liquid chromatography/photodiode array detection for determination of strychnine in blood: A fatal case report," Forensic Science International, vol. 141, no. 1, pp. 17-21, 2004.

[12] K. Wang, Y.-G. Wu, J. Su, J.-J. Zhang, P. Zhang, and X.-M. Qi, "Total glucosides of paeony regulates JAK2/STAT3 activation and macrophage proliferation in diabetic rat kidneys," American Journal of Chinese Medicine, vol. 40, no. 3, pp. 521-536, 2012. 
[13] L. Gu, X. Wang, Z. Liu et al., "A study of Semen Strychniinduced renal injury and herb-herb interaction of Radix Glycyrrhizae extract and/or Rhizoma Ligustici extract on the comparative toxicokinetics of strychnine and brucine in rats," Food and Chemical Toxicology, vol. 68, pp. 226-233, 2014.

[14] Y. Tang, Y. Liu, and X. Liang, "Effect of compatibility of nux vomica and radix paeoniae alba on anti-inflammatory and immune of adjuvant arthritis rats and mechanism analysis," Journal Tradition Chinese Medicine, vol. 33, no. 8, pp. 1010610140, 2014.

[15] Y. Liu, X. Liang, and Y. Tang, "Effect of compatibility of nux vomica and radix paeoniae alba on LD50 and analgesic effect of mice," Journal Tradition Chinese Medicine, vol. 33, no. 8, pp. 660-662, 2014.

[16] D.-Y. He and S.-M. Dai, "Anti-inflammatory and immunomodulatory effects of Paeonia lactiflora Pall., a traditional Chinese herbal medicine," Frontiers in Pharmacology, vol. 2, article 10, 2011.

[17] C. Wang, J. Yuan, H.-X. Wu et al., "Total glucosides of paeony inhibit the inflammatory responses of mice with allergic contact dermatitis by restoring the balanced secretion of pro-/antiinflammatory cytokines," International Immunopharmacology, vol. 24, no. 2, pp. 325-334, 2015.

[18] J. Su, P. Zhang, J.-J. Zhang, X.-M. Qi, Y.-G. Wu, and J.-J. Shen, "Effects of total glucosides of paeony on oxidative stress in the kidney from diabetic rats," Phytomedicine, vol. 17, no. 3-4, pp. 254-260, 2010.

[19] X.-X. Xu, X.-M. Qi, W. Zhang et al., "Effects of total glucosides of paeony on immune regulatory toll-like receptors TLR2 and 4 in the kidney from diabetic rats," Phytomedicine, vol. 21, no. 6, pp. 815-823, 2014.

[20] H. Wu, G. Chen, K. R. Wyburn et al., "TLR4 activation mediates kidney ischemia/reperfusion injury," Journal of Clinical Investigation, vol. 117, no. 10, pp. 2847-2859, 2007.

[21] U. S. Food, "Drug Administration FDA, Guidance for Industry: Bioanalytical Method Validation," in US Department of Health and Human, Services Food and Drug Administration, and Center for Drug Evaluation and Research, USA, 2001.

[22] Y. Zhang, Z. Liu, R. Zhang, P. Hou, K. Bi, and X. Chen, "Nephrotoxicity evaluation of a new cembrane diterpene from Euphorbiae pekinensis Radix with HEK 293T cells and the toxicokinetics study in rats using a sensitive and reliable UFLCMS/MS," Journal of Pharmaceutical and Biomedical Analysis, vol. 119, pp. 159-165, 2016.

[23] D. Galmarini, L. Tarenzi, and G. Cantaluppi, "Acute hepatic failure and renal damage. Experimental and clinical studies," Panminerva, vol. 69, no. 27, pp. 1417-1426, 1978.

[24] A. Jamshidzadeh, R. Heidari, T. Golzar, and A. Derakhshanfar, "Effect of eisenia foetida extract against cisplatin-induced kidney injury in rats," Journal of Dietary Supplements, vol. 13, no. 5, pp. 551-559, 2016.

[25] R. W. Schrier, W. Wang, B. Poole, and A. Mitra, "Acute renal failure: definitions, diagnosis, pathogenesis, and therapy," Journal of Clinical Investigation, vol. 114, no. 1, pp. 5-14, 2004.

[26] S. G. Emeigh Hart, "Assessment of renal injury in vivo," Journal of Pharmacological and Toxicological Methods, vol. 52, no. 1, pp. 30-45, 2005.

[27] C. Liu, Z. Cheng, Y. Wang, X. Dai, J. Zhang, and D. Xue, "Paeoniflorin exerts a nephroprotective effect on concanavalin A-induced damage through inhibition of macrophage infiltration," Diagnostic Pathology, vol. 10, no. 1, article no. 120, 2015.
[28] Y. P. Zhu, "Toxicology of the Chinese herb mu tong (Aristolochia manshuriensis). What history tells us," Adverse Drug React Toxicology Reviews, vol. 21, no. 4, pp. 171-177, 2002.

[29] L. Liu, X.-P. Zhao, D.-F. Zhu, Y.-Y. Cheng, and H.-B. Qu, "Simultaneous LC-MS/MS determination of danshensu and paeoniflorin for permeability studies in Caco-2 intestinal absorption model," Chemical Research in Chinese Universities, vol. 24, no. 4, pp. 420-426, 2008.

[30] C. W. Nogueira, L. P. Borges, and A. C. G. Souza, "Oral administration of diphenyl diselenide potentiates hepatotoxicity induced by carbon tetrachloride in rats," Journal of Applied Toxicology, vol. 29, no. 2, pp. 156-164, 2009.

[31] M. Mishima, Y. Tanimoto, Z. Oguri, and H. Yoshimura, "Metabolism of strychnine in vitro," Drug Metabolism and Disposition, vol. 13, no. 6, pp. 716-721, 1985.

[32] L.-N. Gao, Y. Zhang, Y.-L. Cui, and O. M. Akinyi, "Comparison of paeoniflorin and albiflorin on human CYP3A4 and CYP2D6," Evidence-Based Complementary and Alternative Medicine, vol. 2015, Article ID 470219, 7 pages, 2015.

[33] Y. Wu, K. Ren, C. Liang et al., "Renoprotective effect of total glucosides of paeony (TGP) and its mechanism in experimental diabetes," Journal of Pharmacological Sciences, vol. 109, no. 1, pp. 78-87, 2009.

[34] L. Velíšek, "Models of Chemically-Induced Acute Seizures," Models of Seizures and Epilepsy, pp. 127-152, 2006. 


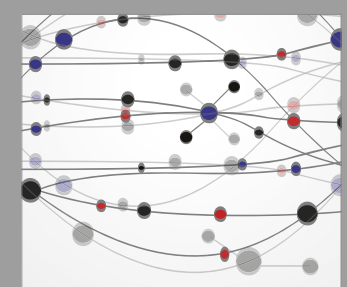

The Scientific World Journal
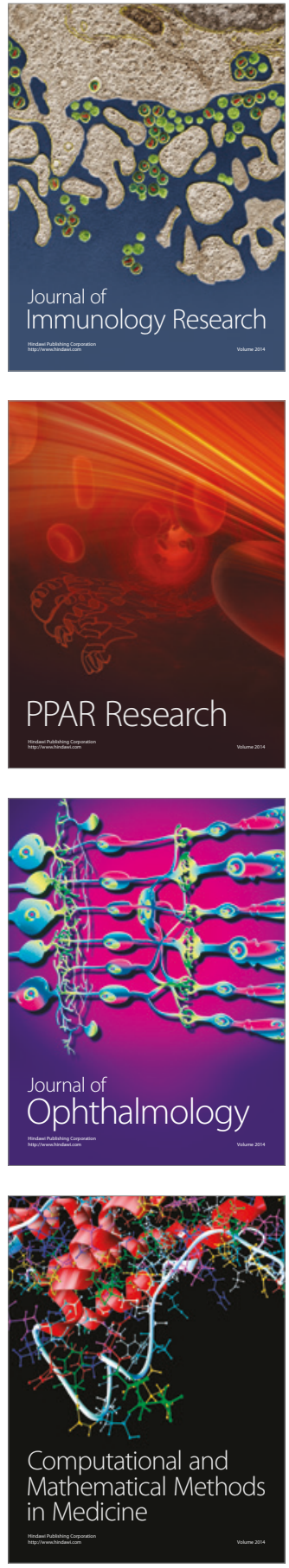

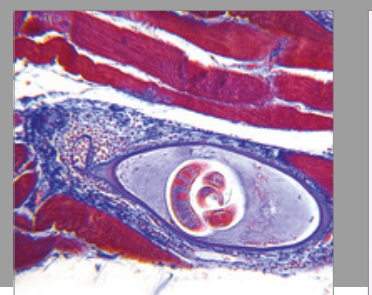

Gastroenterology Research and Practice
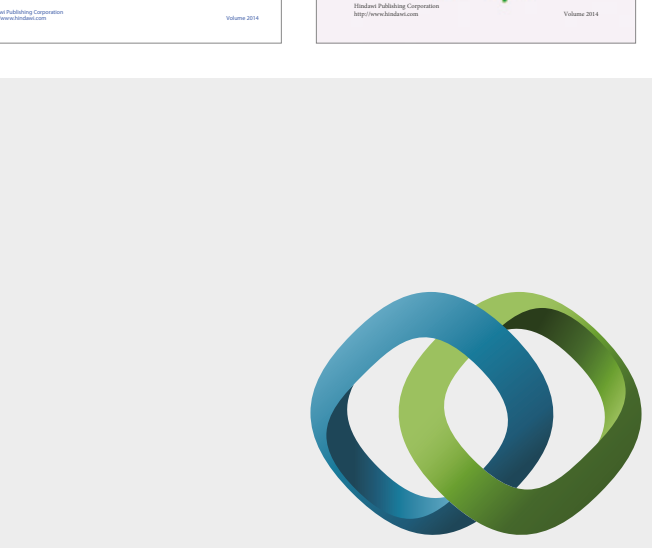

\section{Hindawi}

Submit your manuscripts at

https://www.hindawi.com
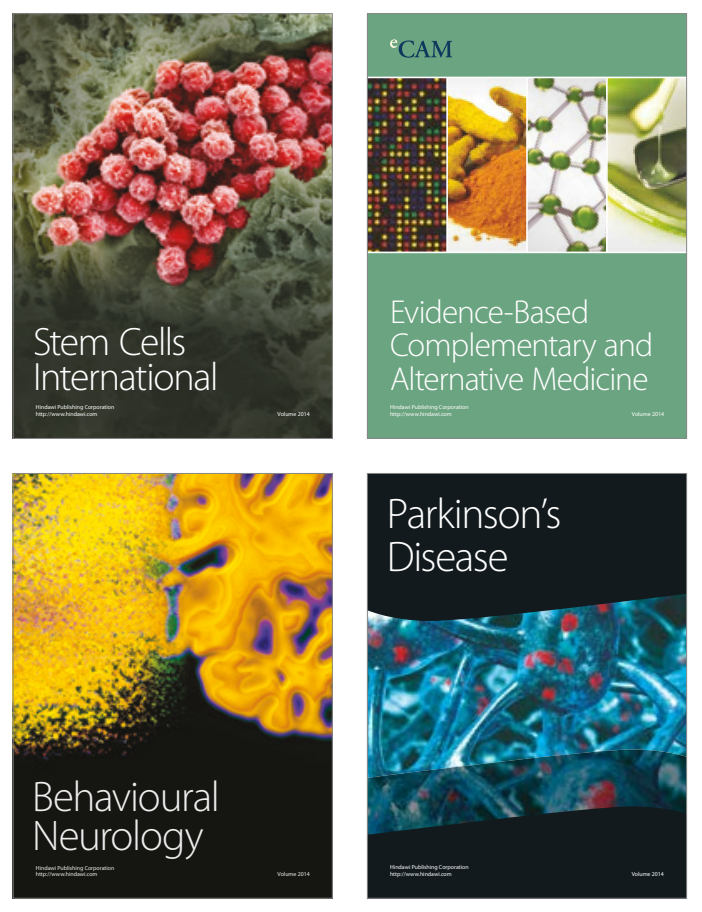
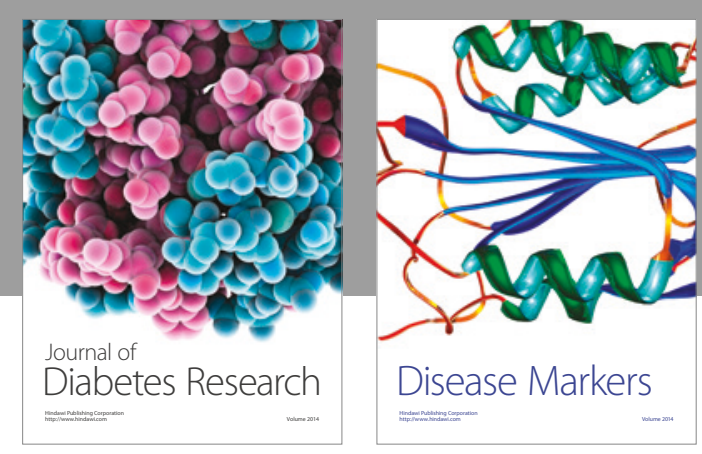

Disease Markers
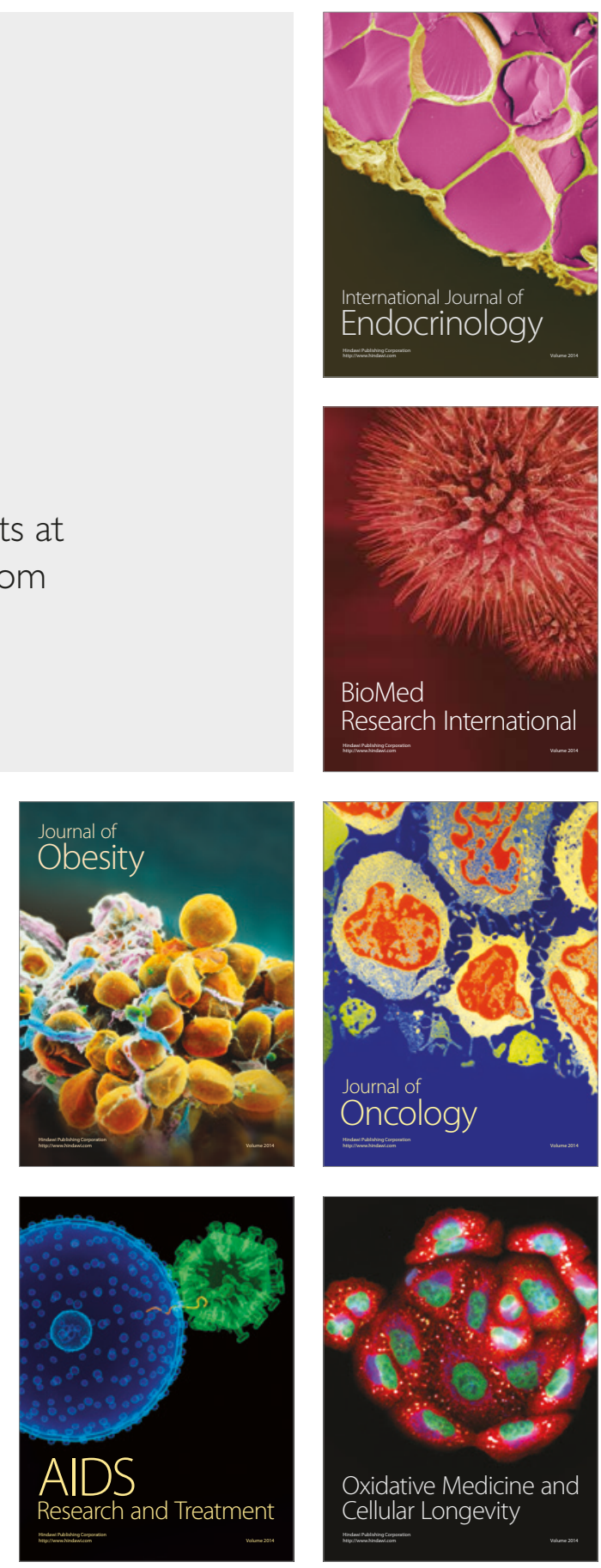